\title{
Optical Network Automation [Invited Tutorial]
}

\author{
Luis Velasco* and Marc Ruiz \\ Optical Communications Group (GCO), Universitat Politècnica de Catalunya (UPC), Barcelona, Spain \\ *e-mail:lvelasco@ac.upc.edu
}

\begin{abstract}
Increased levels of automation will be necessary in view of more stringent performance requirements that next generation optical transport networks need to support in a near term, not only for high capacity, but, even more importantly, for dynamicity, latency, and availability. All these aspects will become more relevant with the growing complexity of modern networks. Network automation targets resource re-optimization to rapidly adapt the network to the expected conditions, quick degradation detection to improve the quality of the connections, as well as failure detection and identification to facilitate maintenance. Network automation requires and implies the collection of data for performance monitoring, being then elaborated by data analytics algorithms to produce meaningful inputs for the network controller, which will finally program the underlying devices. In this paper, we analyze alternative architectures for monitoring and data analytics (MDA) and illustrative control loops are presented aiming at validating the usefulness of MDA to automate optical networks operation.
\end{abstract}

Keywords: Monitoring and data analytics, Autonomic optical networking

\section{INTRODUCTION}

After years of research and development, the Elastic Optical Networking (EON) technology is currently being deployed in optical transport networks. This technology enables among others: $i$ ) the capacity and / or reach increase of optical connections (hereafter, lightpaths) and ii) a finer and dynamic spectrum allocation. The first is enabled by the joint usage of coherent detection, advanced Digital Signal Processing (DSP) techniques, novel modulation formats and soft-decision Forward Error Correction (FEC) codes to recover Bit Error Rate (BER) within the Optical Transponders (TP). The second is possible thanks to programmable Wavelength Selective Switches (WSS) and Reconfigurable Optical Add-Drop Multiplexers (ROADM).

From a network control perspective, an enormous amount of research and standardization effort has been carried out, over the recent years, to implement the Software-Defined Networking (SDN) concept in optical networks. SDN relies on the separation of data and control planes, and leverages programmability and the usage of open interfaces. However, little to no attention has been paid to the operational loop (including monitoring, intelligence and management functionalities), relegating some of them into the Network Management Systems (NMS), and with limited practical operation capabilities. Although EON and SDN technologies can fulfill current capacity and dynamicity requirements, transport networks are expected to support the deployment of upcoming $5 \mathrm{G}$ mobile infrastructures in the near future; $5 \mathrm{G}$ mobile will extend far beyond previous generations and require an enhanced quality of experience for the final users with new services and improved network performance. However, given the increasing network complexity, the main challenge for operators will be to promptly respond to variable network conditions while ensuring full availability and optimization of network resources. Nonetheless, current optical networks are incorporating a complex ecosystem of devices and sensors, which will produce a large amount of data that can be exploited to optimize a network in real-time. To cope with such complex and time-variable scenarios, Machine Learning (ML) - based algorithms [1] are being proposed to facilitate the network operation and predictive maintenance. ML algorithms, fed with real measurements, are able to accurately estimate the Quality of Transmission (QoT) of new lightpaths, to anticipate capacity exhaustion and degradations, or to predict and localize failures, among others (see, e.g., [2], [3]).

In this paper, we present three wide-scope use cases that require MDA-based solutions and whose application will bring clear benefits: $i$ ) network planning and provisioning with reduced margins, $i i$ ) dynamic network adaptation, and iii) lightpath degradation detection and failure localization; Table 1 summarizes the use cases.

\begin{tabular}{|l|l|l|l|}
\hline \multicolumn{4}{|c|}{ Table 1 Target use cases. } \\
\hline $\begin{array}{l}\text { Use case } \\
\text { planning and } \\
\text { provisioning with } \\
\text { reduced margins }\end{array}$ & $\begin{array}{l}\text { Application of just enough } \\
\text { margin in the network } \\
\text { design and in lightpaths } \\
\text { provisioning. }\end{array}$ & $\begin{array}{l}\text { CAPEX saving opportunity } \\
\text { by avoiding or postponing } \\
\text { unnecessary investments at a } \\
\text { given time. }\end{array}$ & $\begin{array}{l}\text { Attenuation, dispersion and other fiber } \\
\text { parameters, the noise figure of } \\
\text { amplifiers, WSS passband, the } \\
\text { sensitivity of TPs, etc. } \\
\text { Those parameters can be used together } \\
\text { with analytical model to estimate } \\
\text { the QoT of lightpaths accurately. } \\
\text { aL-based methods to predict the } \\
\text { probability that the QoT of a candidate } \\
\text { lightpath will not exceed a defined } \\
\text { threshold. }\end{array}$ \\
\hline
\end{tabular}




\begin{tabular}{|c|c|c|c|}
\hline $\begin{array}{l}\text { Dynamic } \\
\text { Network } \\
\text { adaptation }\end{array}$ & $\begin{array}{l}\text { Leveraging on configurable } \\
\text { TPs the allocation of just } \\
\text { enough data rate for any } \\
\text { connection at any time to } \\
\text { cope with traffic dynamics } \\
\text { at minutes or hours scale. }\end{array}$ & 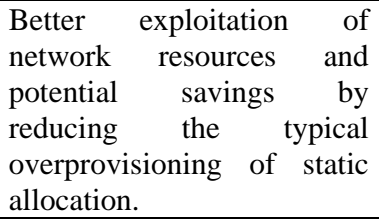 & $\begin{array}{l}\text { Use of models to evaluate the expected } \\
\text { QoT of a lightpath at any new TP } \\
\text { configuration. } \\
\text { Use of models for traffic analysis to } \\
\text { evaluate traffic trends and periodicity. }\end{array}$ \\
\hline $\begin{array}{l}\text { Lightpath } \\
\text { Degradation and } \\
\text { failure } \\
\text { localization }\end{array}$ & $\begin{array}{l}\text { QoT reduces over time due } \\
\text { to network and device } \\
\text { degradation (e.g., fiber cuts } \\
\text { and repairs), ageing, or load } \\
\text { increasing. }\end{array}$ & $\begin{array}{l}\text { Degradation anticipation } \\
\text { allows appropriately tune } \\
\text { systems' parameters before } \\
\text { alarm triggering. } \\
\text { Localizing the element } \\
\text { responsible for a failure } \\
\text { facilitates network } \\
\text { maintenance by planning a } \\
\text { human intervention. }\end{array}$ & $\begin{array}{l}\text { Predictive analysis based on QoT } \\
\text { evolution. } \\
\text { Localization based on the per-system } \\
\text { analysis. Algorithms that find the } \\
\text { potential cause of the failure. }\end{array}$ \\
\hline
\end{tabular}

\section{THE MDA-BASED SYSTEM}

Considering the use cases defined in Table 1, optical devices need to be capable of performing measurements on selected points of the networks, named Observation Points (OP). For example, measurements could be obtained from DSP units within the TPs, as well as from specific monitoring devices installed within the network. Specifically, DSPs can provide measurements or estimations of power levels, fiber channel characteristics (e.g., accumulated dispersion, fiber nonlinear coefficient, polarization mode dispersion) and QoT-related parameters (e.g., linear OSNR and BER). Furthermore, monitoring devices, like cost-effective optical spectrum analyzers (OSA) and optical time-domain reflectometer placed at predefined locations of the network, can provide specific measurements of optical signals and fiber segments.

It is also important to consider the target accuracy of data analytics to define the sufficient amount of data to be collected and stored, as the accuracy depends on the amount of data that is considered in the MDA system for decision making. For example, if a system would operate in pure linear regime, the pre-FEC BER could be enough to estimate the actual OSNR and then the relative system margin. However, real networks do not always operate in a full linear regime, and therefore, the pre-FEC BER may result in being unsuitable to always provide an accurate prediction of the instantaneous OSNR margin. Consequently, enough data need to be stored to achieve a predefined accuracy, especially under low or zero-margin network operation.

Another key factor is the update frequency; an instantaneous collection of monitoring data could produce negative effects, so it is important to determine the right frequency for data collection. For instance, once a lightpath is established, and until there are no substantial changes in the network, there is no need to update the fiber channel values. Contrarily, parameters such as amplifier power levels require a higher update frequency, although old values could be discarded if the individual amplifier works properly. Overall, all these data will be ultimately used by the MDA system, which might also incur in saturation or in drawing suboptimal decision in case of overwhelming or contradictory data.

Different strategies can be envisioned to solve this issue: $i)$ using thresholds, which are simple but inaccurate; ii) experience and physical knowledge, which could lead to evaluation errors in case of not

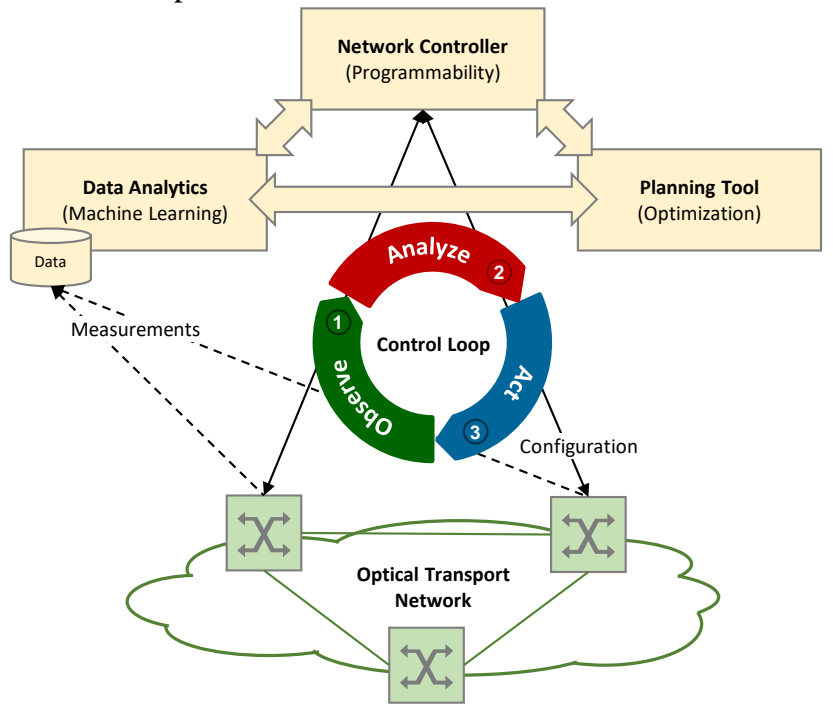

Fig. 1 MDA enable OAA control loop implementation. predicted scenarios; $\mathrm{iii}$ ) by designing an intelligent MDA system that can decide based on physical conditions what data should be analyzed and consider possible dependencies.

Finally, it is worth pointing out that the main challenge (and limitations) occur in multi-vendor scenarios. In this context, a proactive MDA system could anticipate issues before they happen and issue the proper recommendations provided that the MDA system is aware of the configuration of all involved nodes at any time.

In conclusion, the opportunities that MDA opens go far beyond a monitoring data collector and storage platform. The analysis of the collected data can discover knowledge and use it to proactively self-configure and self-tune the network in a cost-effective (near) real-time manner by adapting resources to future conditions. Therefore, thanks to the application of data analytics to monitored data, observe-analyze-act control loops can be enabled, where outcomes of such analysis can be used for event notifications together with recommended actions to the 
SDN controller (Fig. 1). Last but not least, useful models can be estimated from monitoring data to feed planning tools in order to compute optimal solutions for the expected future conditions.

\section{MDA ARCHITECTURES}

In this section, we present and analyze several architectural approaches to bring real MDA capabilities to the network (see Fig. 2). Specifically, three architectures are considered depending on where data analytic capabilities are enabled, namely: $i$ ) centralized, ii) distributed, and iii) hierarchical.

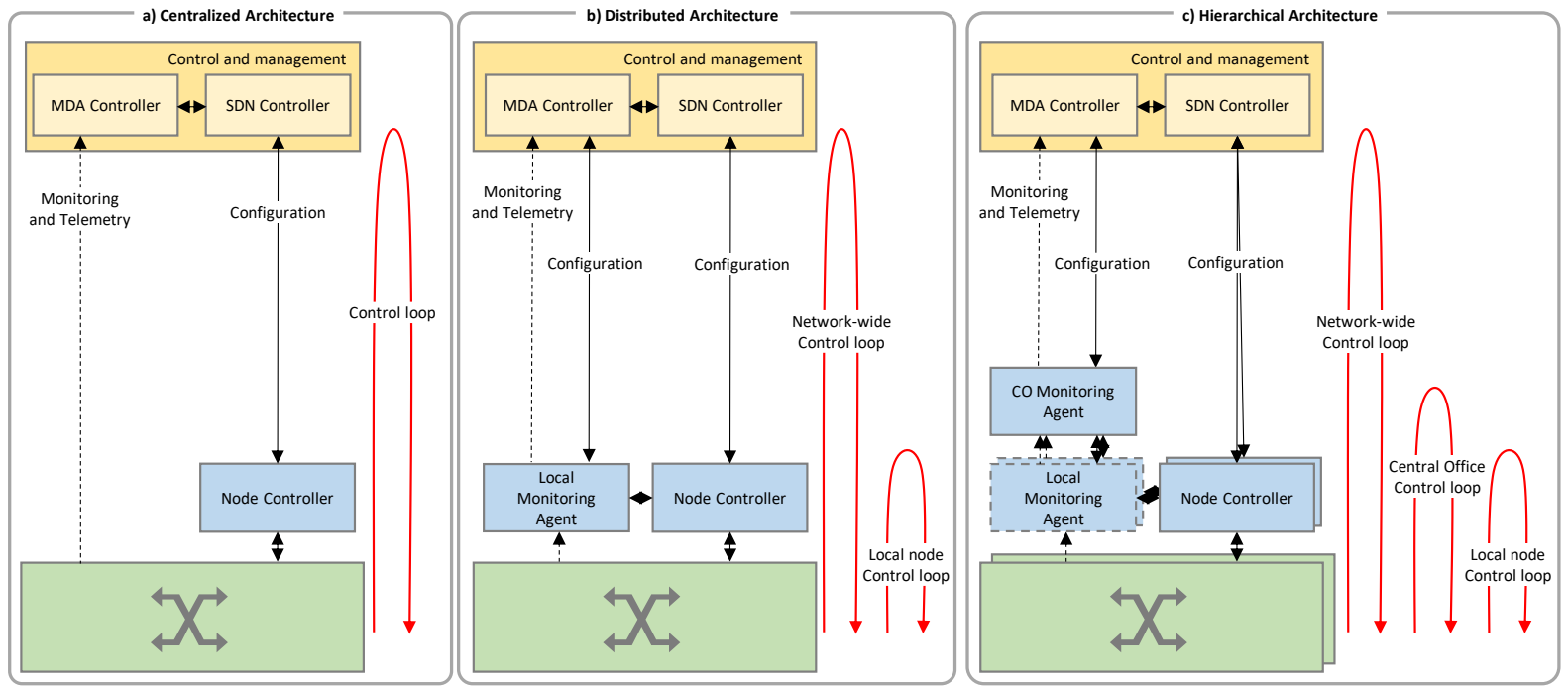

Fig. 2 Overview of the centralized (a), distributed (b), and hierarchical (c) MDA architectures.

The centralized architecture (Fig. 2a) consists in detaching the monitoring repository and the data analytics system, if any, from the NMS to create a separate specific centralized MDA controller that can interface the SDN controller and other systems within the control plane (see, e.g., Ciena Blue Planet). To keep the MDA architecture simple, let us consider that its only mission is to expose an interface to collect monitoring and telemetry data from the network devices. Measurements are stored in a (big data) repository, and data analytics algorithms can be devised to discover knowledge to be used to predict and/or to detect anomalies and degradations before they negatively impact on the network performance. Such predicted events can be notified to the SDN controller together and include a recommended action to guide the SDN controller; the recommended action is a suggestion that the SDN controller can follow or ignore and apply its own policies. As an example, in some cases BER degradation can be predicted ahead of time in a lightpath before any threshold is exceeded by analyzing the BER evolution as measured at the receiver; this is notified to the SDN controller together with a recommended action after analyzing several alternatives, including change of the modulation format (also via probabilistic shaping), reroute of the lightpath (e.g., to avoid some links); or also to increase, if possible, the amount of overhead used by the FEC. The notification to the SDN controller might trigger a re-configuration, hence closing the loop and adapting the network to the new conditions.

The centralized MDA architecture presents some limitations; for instance, the time to detect an anomaly or degradation is related to the update frequency. Therefore, to reduce the detection times, the amount of data to be conveyed to the MDA controller needs to be increased accordingly. Another issue is related to the control of monitoring; specifically, to activate telemetry on-demand once an event has been detected.

To overcome these problems, the distributed architecture (Fig. 2b) includes MDA agents in charge of collecting measurements from a single node, while keeping the MDA controller centralized [4]-[5]. The MDA agent exposes two unified interfaces toward the MDA controller for collecting data and monitoring configuration; in addition, specific interfaces for data collection and monitoring control allow the MDA agent connecting with the network device. The data analytics capabilities deployed close to the network nodes enable local control loops implementation; measurements can be analyzed locally, and configuration can be tuned and adapted to changing conditions. However, the co-existence of two controllers, the SDN and the MDA, in charge of configuring network devices, might create conflicts, so it would be desirable to clearly separate responsibilities among them.

The distributed architecture includes a dedicated MDA agent for every node in the network, which might present some limitations when disaggregated optical network nodes (e.g., TPs and ROADMs) and monitoring devices are deployed within the same central office (CO) [5]. For this reason, the hierarchical architecture (Fig. 2c) includes a per-CO MDA agent that collects measurements from every network device in the $\mathrm{CO}$ and exposes a single set of interfaces toward the MDA controller. In this case, measurements from one device can be analyzed in the $\mathrm{CO}$ MDA agent and configuration can be tuned to another device within the same $\mathrm{CO}$, thus minimizing the intervention of the MDA controller. The per-CO MDA agent could (or not) replace every node MDA agent thus, reducing systems count. 
The strengths and weaknesses of the analyzed MDA architectures are summarized in Table 2, where the features of each architecture include those of the previous.

Table 2 Strengths and weaknesses of several monitoring and data analytics architectures.

\begin{tabular}{|c|c|c|c|}
\hline Architecture & Features & Strengths & Weaknesses \\
\hline Centralized & $\begin{array}{l}\text { - Includes a centralized } \\
\text { MDA system with a data } \\
\text { repository for monitoring/ } \\
\text { telemetry data where data } \\
\text { analytics can be applied. } \\
\text { - Monitoring and telemetry } \\
\text { activation and deactivation } \\
\text { is managed by an external } \\
\text { system, e.g., the NMS. }\end{array}$ & $\begin{array}{l}\text { - Data analytics results can be used for } \\
\text { network self-adaptation to changing } \\
\text { conditions. } \\
\text { - Interfaces with the SDN controller } \\
\text { and NFV orchestrator can be easily } \\
\text { standardized. }\end{array}$ & $\begin{array}{l}\text { - Different monitoring / telemetry } \\
\text { protocols need to be available at } \\
\text { the MDA controller. } \\
\text { - The amount of data to be collated } \\
\text { from the nodes increases } \\
\text { exponentially to keep low } \\
\text { reaction times against anomalies } \\
\text { or degradations. } \\
\text { - Configuration tuning is not } \\
\text { supported. } \\
\text { - Network slicing is difficult to be } \\
\text { supported. }\end{array}$ \\
\hline Distributed & $\begin{array}{l}\text { - Allows data analytics to be } \\
\text { applied within the MDA } \\
\text { agents, close to the } \\
\text { network nodes. Control } \\
\text { loops can be implemented } \\
\text { locally at the node level. } \\
\text { - Monitoring and telemetry } \\
\text { activation / deactivation is } \\
\text { managed by the MDA } \\
\text { controller. }\end{array}$ & $\begin{array}{l}\text { - Supports configuration tuning [6]. } \\
\text { - It reduces data to be conveyed to the } \\
\text { MDA controller since patter } \\
\text { recognition can be done in the MDA } \\
\text { agents. } \\
\text { - MDA agents expose one single } \\
\text { monitoring and telemetry interface to } \\
\text { the MDA controller. } \\
\text { - Supports network slicing [4]. }\end{array}$ & $\begin{array}{l}\text { - A configuration interface needs to } \\
\text { be defined between the MDA } \\
\text { controller and the agents. } \\
\text { - More complex MDA controller as } \\
\text { more features are added, like } \\
\text { monitoring and telemetry control, } \\
\text { and configuration tuning. } \\
\text { - CO control loops are not } \\
\text { supported. }\end{array}$ \\
\hline Hierarchical & $\begin{array}{l}\text { - It includes a per-CO MDA } \\
\text { agent that connects to all } \\
\text { the nodes in the CO. }\end{array}$ & $\begin{array}{l}\text { - Control loops can be implemented } \\
\text { locally at the node, as well as at the } \\
\text { CO level involving more than one } \\
\text { node. } \\
\text { - Appropriate for node disaggregation } \\
\text { scenarios, where monitoring devices } \\
\text { can be installed in one node, but } \\
\text { configuration tuning needs to be done } \\
\text { in a different node [5]. } \\
\text { - It reduces the total number of agents } \\
\text { and the number of interfaces toward } \\
\text { the MDA controller. }\end{array}$ & $\begin{array}{l}\text { - Requires more complex MDA } \\
\text { agents to consider complex } \\
\text { relations among nodes. }\end{array}$ \\
\hline
\end{tabular}

\section{SUMMARY}

We have provided an overview of optical network infrastructure automation, key requirements and current enabling optical technologies. The role of MDA in optical networking has been studied through three wide-scope use cases covering the main network operations: $i$ ) network planning and provisioning, ii) dynamic network adaptation, and iii) degradation detection and failure localization, where clear benefits have been unveiled. Interestingly, current networking devices are already capable of performing measurements that support those use cases. Additional data can be collected by installing specific monitoring devices at predefined locations.

\section{ACKNOWLEDGEMENTS}

The research leading to these results has received funding from the Spanish MINECO TWINS project (TEC201790097-R), and from the Catalan Institution for Research and Advanced Studies (ICREA).

\section{REFERENCES}

[1] D. Rafique and L. Velasco, "Machine Learning for Optical Network Automation: Overview, Architecture and Applications," IEEE/OSA Journal of Optical Communications and Networking, vol. 10, pp. D126-D143, 2018.

[2] A. P. Vela et al., "BER Degradation Detection and Failure Identification in Elastic Optical Networks," IEEE/OSA Journal of Lightwave Technology, vol. 35, pp. 4595-4604, 2017.

[3] A. P. Vela et al., "Soft Failure Localization during Commissioning Testing and Lightpath Operation," IEEE/OSA Journal of Optical Communications and Networking, vol. 10, pp. A27-A36, 2018.

[4] L. Velasco et al., "An Architecture to Support Autonomic Slice Networking [Invited]," IEEE/OSA Journal of Lightwave Technology, vol. 36, pp. 135-141, 2018.

[5] L. Velasco et al., "Building Autonomic Optical Whitebox-based Networks," IEEE/OSA Journal of Lightwave Technology, vol. 36, pp. 3097-3104, 2018.

[6] Ll. Gifre et al., "Autonomic Disaggregated Multilayer Networking," IEEE/OSA Journal of Optical Communications and Networking, vol. 10, pp. 482-492, 2018. 